Naohiko Seki • Takanori Azuma - Tsutomu Yoshikawa

Yasuhiko Masuho • Masa-aki Muramatsu

Toshiyuki Saito

\title{
cDNA cloning of a new member of the Ras superfamily, RAB9-like, on the human chromosome Xq22.1-q22.3 region
}

\begin{abstract}
Members of the RAB protein family regulate vesicular trafficking and reside in specific intercellular compartments. A new member of the RAB family was identified through a public database search, and its fulllength cDNA was isolated from a human fetal brain cDNA library. The predicted protein product of the gene consists of 201 amino acid residues, and the protein has $86 \%$ similarity to human RAB9 at the amino acid level. We designated the new gene RAB9-like. Northern blot analysis showed that the gene was transcribed ubiquitously in various human tissues. A database search revealed that the gene is divided into three exons and spans approximately $7.2 \mathrm{~kb}$ of the genome DNA of chromosome Xq22.1-q22.3 region.
\end{abstract}

Key words RAS superfamily of small GTP-binding proteins $\cdot \mathrm{RAB} \cdot \mathrm{RAB} 9 \cdot$ Chromosome Xq22.1-q22.3

\section{Introduction}

The RAS superfamily of small guanosine triphosphate (GTP)-binding proteins, which includes the Ras, Ral, Rho, Rap, and Rab families, is involved in controlling a diverse set of essential cellular functions. The RAB family of small $\mathrm{G}$ proteins, consisting of more than 40 members, regulates intercellular vesicle trafficking, including exocytosis, endocytosis, and recycling (Nuoffer and Balch 1994; Novick

N. Seki $(\bowtie) \cdot$ T. Azuma · T. Yoshikawa · Y. Masuho

M. Muramatsu

Helix Research Institute, 1532-3 Yana, Kisarazu, Chiba 292-0812,

Japan

Tel. +81-438-52-3964; Fax +81-438-52-3952

e-mail: nseki@hri.co.jp

N. Seki · T. Saito

Genome Research Group, National Institute of Radiological

Sciences, Chiba, Japan

The nucleotide sequence data reported in this paper have been deposited to DDBJ, EMBL, and GenBank database under the accession number AB036693. and Zerial 1997; Chavrier and Goud 1999; Gonzalez and Scheller 1999). The mammalian RAB proteins show striking similarities to Saccharomyes cerevisiae YPT1 and SEC4 proteins, and encode Ras-related GTP-binding proteins involved in the regulation of secretion. Initially, several human RAB cDNAs were isolated from a human pheochromocytoma library with a probe derived from the SEC4 gene (Zahraoui et al. 1989).

Canine Rab9 was first identified in a screen for YPT1 and SEC4 protein-related cDNA clones (Chavrier et al. 1990). The sequence of the full-length protein showed $37 \%$ homology to YPT1 protein (Schmitt et al. 1986). Rab9 has been localized to components of the endocytic/exocytic pathway. It has been implicated in the recycling of membrane receptors, such as the mannose 6-phosphate receptor from early endosomes to the trans Golgi network (Chavrier et al. 1990; Lombardi et al. 1993; Shapiro et al. 1993; Riederer et al. 1994). In humans, a cDNA for $R A B 9$ was isolated from human U937 cells, using a reverse transcriptase-polymerase chain reaction (RT-PCR) method; its amino acid sequence is almost $98 \%$ identical to that of canine Rab9 (Davies et al. 1997). While an antisense inhibition of Rab9 proteins in human fibroblast cells caused severe cell vacuolation resembling that of cells from a patient with Chediak-Higashi syndrome (Davies et al. 1997), the precise functions of Rabs are still poorly understood.

To date, the family is still expanding, with the use of PCR-based cloning approaches employing highly conserved sequence stretches of the RAB proteins. Here we report the sequence features, expression profile, and chromosomal assignment of a novel gene which has high similarity to the human $R A B 9$ gene.

\section{Isolation and source of human RAB9-like gene}

Recently, we have systematically isolated cDNAs for novel RAB family genes, human $R A B 26$-related (Seki et al. 2000) (accession number, AB027137), human RAB23 (accession number, AB034244), and mouse Rab9 (accession number, 

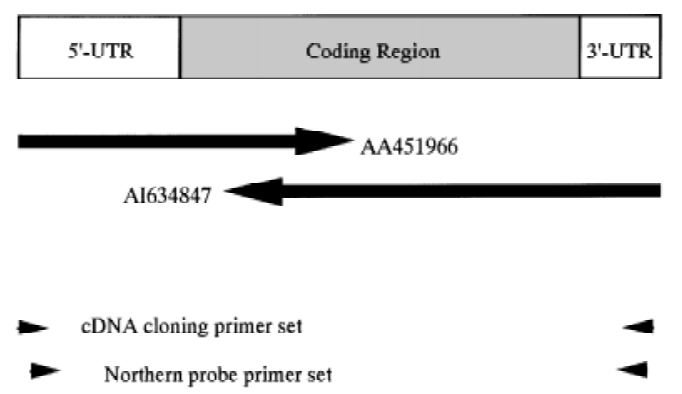

RH mapping primer set

A cgagcggcacgtgtcaggccaccgaggtccaagccgcacttgctgccccattgaggacga ggaggcagcaggagcagtgacggtgactctaaggagccggattcccggcacgcagagctg acctgcctggcacccgcggccetctcctgtttccttcccattgtgttggcaccctaaaa gaaagaataaaacaacaacaggaaaaaaggaaaatatttaaattgtgacaaaacccac tgggttctcttggttacaaactccttcccttctggtgctgcaaaaATGAGTGGGAAATCC $M \quad S \quad G \quad K \quad S$

CTGCTCTTAAAGGTCATTCTCTTGGGTGATGGTGGAGTTGGGAAAAGTTCGCTTATGAAC

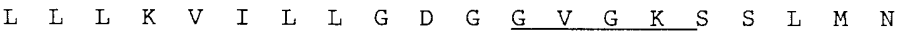
CGTTACGTAACCAACAAATTTGACTCCCAGGCTTTTCACACCATAGGGTAGAGTTCTTA $\begin{array}{llllllllllllllllllll}R & \mathrm{Y} & \mathrm{V} & \mathrm{T} & \mathrm{N} & \mathrm{K} & \mathrm{F} & \mathrm{D} & \mathrm{S} & \mathrm{O} & \mathrm{A} & \mathrm{F} & \mathrm{H} & \mathrm{T} & \mathrm{I} & \mathrm{G} & \mathrm{V} & \mathrm{E} & \mathrm{F} & \mathrm{L}\end{array}$ AATCGAGATCTGGAGGTAGATGGACGCT'TTGTAACCCTCCAGATCTGGGACACTGCAGGG \begin{tabular}{llllllllllllllllllll}
$N$ & $R$ & $D$ & $L$ & $E$ & $V$ & $D$ & $G$ & $R$ & $F$ & $V$ & T & I & $Q$ & I & W & D & T & A & $G$ \\
\hline
\end{tabular} CAGGAACGTTTCAAGAGCCTTAGGACACCCTTCTACAGGGGAGCAGACTGCTGCCTCTTG $\begin{array}{llllllllllllllllllllll}Q & E & R & F & K & S & L & R & T & P & F & Y & R & G & A & D & C & C & L & L\end{array}$ ACCTTCAGCGTGGATGATCGGCAGAGCTTCGAGAATCTTGGTAACTGGCAGAAAGAATTT $\begin{array}{llllllllllllllllllllllll}T & F & S & V & D & D & R & Q & S & F & E & N & L & G & N & W & Q & K & E & F\end{array}$ ATTTACTATGCGGATGTGAAGGACCCTGAGCATTTCCCCTTTGTAGTTCTGGGTAACAAG \begin{tabular}{lllllllllllllllllllll}
$I$ & $Y$ & $Y$ & $A$ & $D$ & $V$ & $K$ & $D$ & $P$ & $E$ & $H$ & $F$ & $P$ & $F$ & $V$ & $V$ & $L$ & $G$ & $N$ & $K$ \\
\hline
\end{tabular} GTAGACAAAGAGGATAGGCAAGTGACTACTGAGGAGGCACAAACCTGGTGCATGGAGAAT $\begin{array}{lllllllllllllllllllllll}V & D & K & E & D & R & Q & V & T & T & E & E & A & Q & T & W & C & M & E & N\end{array}$ GGGGATTACCCTTATTTAGAAACTAGTGCCAAAGATGATACTAATGTGACAGTGGCCTTT

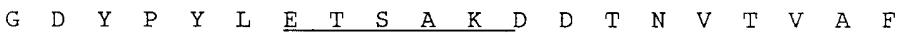
GAAGAAGCTGTCAGGCAGGTGCTGGCTGTAGAGGAACAGCTGGAGCATTGCATGTTGGGT

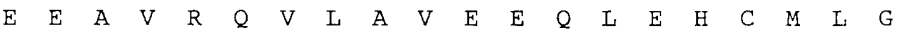
CACACCATTGACTTGAACAGTGGCTCCAAAGCAGGGTCTTCGTGCTGTTAAagataggga $\begin{array}{llllllllllllllllllllllllll}H & T & I & D & L & N & S & G & S & K & A & G & S & S & C & C & *\end{array}$ gccttttaaaatgtgccccaaattgatcagtcagtagtgtaagaataactgtgcccctc taagagtgcacacacacgcacacaagagggtaagagacaaggttctgattgtgaaacaga gccttcaaattgaagtgtagattgattt
Fig. 1. A Schematic representation of the relationship between the $R A B$ 9-like gene and the two expressed sequence tag (EST) sequences. The polymerase chain reaction (PCR) primer sets are shown below the representation. UTR, Untranslated region; $R H$, Radiation hybrid. B Nucleotide sequence and deduced amino acid sequence of the $R A B 9$ - like gene. Asterisk denotes the termination codon. The nucleotide sequence data reported here will appear in the DDBJ, EMBL, and GenBank nucleotide sequence databases with the accession number AB036693. The conserved regions of the amino acid sequence involved in guanosine triphosphate (GTP) binding are underlined
AB027290), using a predicted gene database, designated as Virtual Transcribed Sequence (VTS) (Miyajima et al. 2000). Using a cDNA sequence of mouse Rab 9 gene (accession number, AB027290) as a query sequence, we searched for the novel human RAB-related sequence in the public human expressed sequence tag (EST) database, using the tBLASTN program (http://www.ncbi.nlm.nih.gov/ cgi-bin/BLAST/nph-newblast?Jform=1), and found two ESTs (accession numbers, AI634847 and AA451966) with a high similarity sequence with the human $R A B 9$ gene (accession numbers, U44103), but not identical. Then, we performed PCR, using specific primers designed from the two EST sequences, to obtain a cDNA containing the putative complete coding sequence. Primers used for the cDNA cloning were 5'-CGA GCG GCA CGT GTC AGG CCA CCG AGG-3', corresponding to nucleotides 1 to 27 , and $5^{\prime}$ AAA TCA ATC TAC ACT TCA ATT TGA AAG-3', corresponding to nucleotides 1026 to 1049 (Fig. 1A). A single-sized RT-PCR product was obtained from human brain RNA, showing that the sequence is really transcribed. The RT-PCR band was cloned into TA cloning vector (Invitrogen, Carlsbad, CA, USA), and several cDNA clones were subjected to sequencing to eliminate possible artificial mutations by PCR. The structural relationship between the determined cDNA and the two EST sequences, AI634847 and AA451966, is shown in Fig. 1A.

The nucleotide sequences of both strands were deter- mined by the primer walking method, using an ABI377 sequencer (Perkin Elmer, Norwalk, CT, USA) according to the supplier's instructions. The isolated cDNA clones were $1049 \mathrm{bp}$ in length and contained an open reading frame for a predicted protein of 201 amino acid residues with a calculated molecular weight of approximately 37.7 kiloDaltons (Fig. 1B). The typical polyadenylation signal was not observed in the $3^{\prime}$ untranslated sequence. However, the present sequence should contain the entire proteincoding sequence, referring the other family proteins as follows and in-frame termination stop codon near its $3^{\prime}$ terminus.

Homology search for relevant amino acid sequences in the protein database (NCBI Protein Database and Swiss Protein Database) revealed that the protein was most homologous to human RAB9 (accession number, U44103), having $76 \%$ identity and $86 \%$ similarity at the amino acid level. Human RAB7 (accession number, U44104) and the identified protein showed $53 \%$ identity and $67 \%$ similarity at the amino acid level (Fig. 2). We designated the gene, RAB9-like. The nucleotide sequence of the human RAB9like gene will appear in GenBank/EMBL/DDBJ databases under the accession number, AB036693. Sequence analysis and comparison showed that the RAB9-like gene is clearly a low-molecular wight GTP-binding protein of the Rab family, containing the conserved GTP-binding regions known to be present in this group (Fig. 1B). 
RAB $9-1$ i ke

RA B 9

RAB 7
MSGKSLLLKV I LLG DG GVGKSSLMNRYVTNKF DSQAFHT I GVE F LNRDLEVDGRFVTLQ MAG KSSLFKV I LLG DGGVGKS LMNR YVTNKF DTQLFHT I GVEF LNKDLEVDGHFVTMQ MTSRKKVLLKVITLG DSGVGKTS LMNQYVNKK FSNQYKAT I GA DF LTKEVMVDDRLVTMQ

IWDTAGQER FKSLRT PFYRGADCCLLTFSVDDRQSFENLGNWQK F I Y YADVKDPEHFPF IWDTAGQERFRSLRT F YRGSDC CLLTFSVDDSQSFQN LSNWKKE F I Y YADVKEPESF P F IWDTAGQERFQSLGVAFY RGADC CVLVFDVTA P TFKTLDSWRDEFLVQAS P RDP ENF P F

VVLGNKVDKEDRQVTTEEAQTWCMENG DY YLETSAKDDTNVTVAFEEAVRQVLAVEEQL VILGNKIDISERQVSTEEAQAWCRDNG DY Y FTSAKDATNVAAAF E EAVRVLATEDRS VVLGNKVDLENRQVATKRAQAWCYSKNN IPY FTSAKEAINVEQAFQT I ARNALKQETEV

RA B 9

RAB 7

RAB 9 - 1 ike

RA B 9

E. . HCMLGHTIDLNSG SKAGSSCC*

D. . .HLIQTDTVNLHRKPKPSSSCC *

ELYNEF PEP IKLDKNDRAKASAESCSC *
Fig. 2. Alignment of human RAB9-like (accession number, $\mathrm{AB} 036693$ ), human $R A B 9$ (accession number, U44103), and human $R A B 7$ (accession number, U44104) genes. Identities are indicated by black background and similar residues are shadowed. Asterisks denote termination codons
Fig. 3. Northern blot analysis of human RAB9-like gene. Northern blot filters containing adult human poly $(\mathrm{A})^{+}$RNAs $(2 \mu \mathrm{g} / \mathrm{lane})$ were purchased from Clontech Laboratories (Palo Alto, CA, USA), and hybridization and washing were performed following the manufacturer's instructions. The 961-bp cDNA fragment containing the entire open reading frame was labelled with $\left[\alpha-{ }^{32} \mathrm{P}\right] \mathrm{dCTP}$ and used as a hybridization probe. Size markers $(l e f t)$ are in kilobases

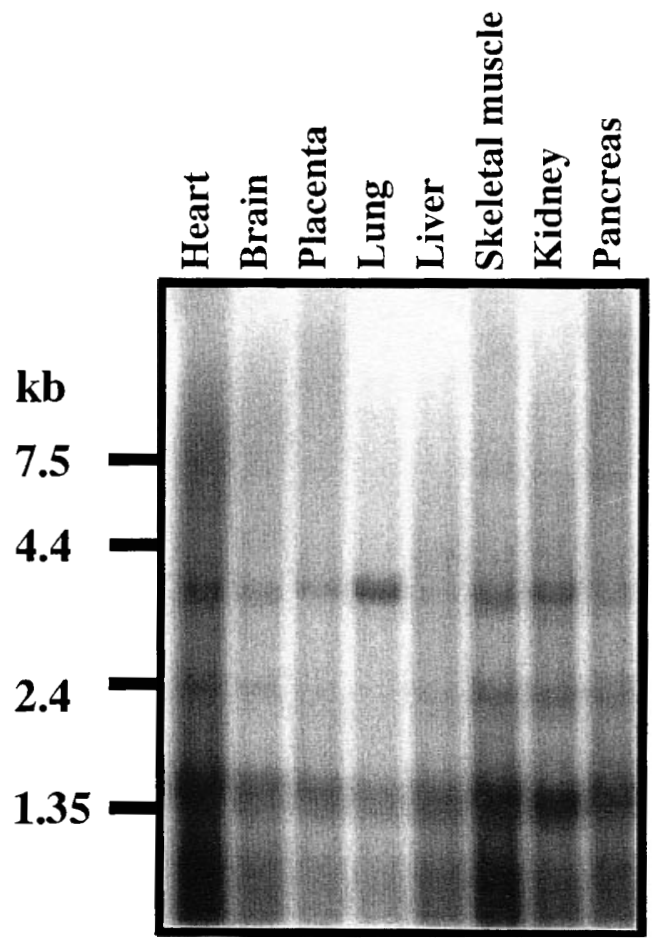

\section{Expression profile of RAB9-like gene}

We examined the distribution of human RAB9-like transcript in various human tissues by Northern blot analysis, as described previously (Seki et al. 1998). The 961-bp cDNA fragment containing the entire open reading frame was used as a hybridization probe. Primers used for the probe preparation were $5^{\prime}$-ACT TGC TGC CCC ATT GAG GAC-3', corresponding to nucleotides 38 to 58 , and $5^{\prime}$-GTC TCT TAC CCT CTT GTG TGC-3', corresponding to nucleotides 978 to 998 . A major band of $1.4 \mathrm{~kb}$ was observed, and two minor bands, of approximately 2.3 and $4.0 \mathrm{~kb}$, were also detected (Fig. 3). The 1.4-kb size of the major band is consistent with the notion that our clone is full-length. The 1.4- $\mathrm{kb}$ signal was detected in all the tissues examined, suggesting that the RAB9-like gene described in the present study may be involved in the basic housekeeping function of cells. It is not known whether the longer signals were derived from alternative form(s) of RAB9-like mRNA or another related gene transcript(s), and this remains to be elucidated.

\section{Genomic structure and chromosome mapping of the RAB9-like gene}

The exon-intron boundaries of the human RAB9-like gene were determined by aligning the cDNA sequence with the genomic sequence (accession numbers, AL035553; 
Table 1. Intron-exon boundaries of the RAB9-like gene

\begin{tabular}{ccll}
\hline Exon no. & Exon size $^{\mathrm{a}}$ & Splice acceptor $^{\mathrm{b}}$ & Splice donor $^{\mathrm{b}}$ \\
\hline 1 & 169 & & ATTGTGTTGG gtaggtgcca \\
2 & 74 & gttttatag CACCCTAAAA & CTAAAAATGG gtaggtattt \\
3 & 806 & ttatttcag GTTCTCTTGG & \\
\hline
\end{tabular}

Intron-exon junctions were established by comparison of cDNA and genomic sequences

${ }^{\text {a }}$ Size in base pairs

${ }^{\mathrm{b}}$ Sequences at the splice junction. Exonic sequences are shown in capital letters, with intronic sequences

shown in lowercase letters. Invariant nucleotides (ag/gt) are in boldface type

clone RP4-540A13). As summarized in Table 1, all the splicing sites conformed to the ag-gt rule, in that there are always ag and gt dinucleotides at the splice acceptor and donor sites, respectively. The RAB9-like gene was divided into three exons, the lengths of which were: $169 \mathrm{bp}$ (exon 1), $74 \mathrm{bp}$ (exon 2), and 806bp (exon 3), and spanned approximately $7.2 \mathrm{~kb}$ of the genome DNA. Exon 3 contained the ATG codon for the initial methionine and TAG termination.

Chromosomal assignment of the human RAB9-like gene was done by PCR analysis of a radiation hybrid panel (Genebridge4; Research Genetics, Huntsville, AL, USA), as described previously (Seki et al. 1999, 2000). The human RAB9-like-specific PCR primers, (5'-CAC ACC ATT GAC TTG AAC AGT-3', corresponding to nucleotides 841 to 861 , and 5'-CTT ACC CTC TTG TGT GCG TGT$3^{\prime}$, corresponding to nucleotides 974 to 994 ), gave rise to an amplified product with a size of $154 \mathrm{bp}$ by genomic PCR. Statistical analysis of the radiation hybrid data was performed using the RHMAPPER software package (http:// carbon.wi.mit.edu:8000/cgi-bin/contig/rhmapper.pl). The data vector for the human $R A B$ 9-like gene was 1000000100 $00000011110000000000 \quad 0110000000 \quad 0100000000$ 0000010000010001000001010000000002101100200 and the consequent report indicated that the gene was placed to $4.08 \mathrm{cR}$ distal from the marker AFMA275ZC9 (lod > 3.0). The region including the marker was cytogenetically mapped to the Xq22 region. On the other hand, the UniGene database (http://www.ncbi.nlm.nih.gov/UniGene/) has noted that the human $R A B 9$ gene was mapped to the XpTEL-DXS1061 region. The chromosomal positions of the $R A B$ 9-like and $R A B 9$ genes are indicated in Fig. 4.

Vesicular transport to and from the lysosome and late endosome is defective in patients with Chediak-Higashi syndrome, and in mutant beige mice (Barbosa et al. 1996, 1997). In Chediak-Higashi syndrome, and in mutant beige mice, cells have giant, perinuclear vesicles with characteristics of late endosomes and lysosomes that arise from dysregulated homotypic fusion (Brandt et al. 1975; Willingham et al. 1981; Burkhardt et al. 1993). Antisense expression of Rab9 produced cells that contained numerous large vacuoles, a phenotype resembling that of fibroblasts from patients with Chediak-Higashi syndrome (Davies et al. 1997). Although the recently identified responsible genes in Chediak-Higashi syndrome and beige mutation do not code any Rab proteins, the above antisense experiment suggests that Rab proteins may be involved in the same biochemical pathway in which the disease genes take part.

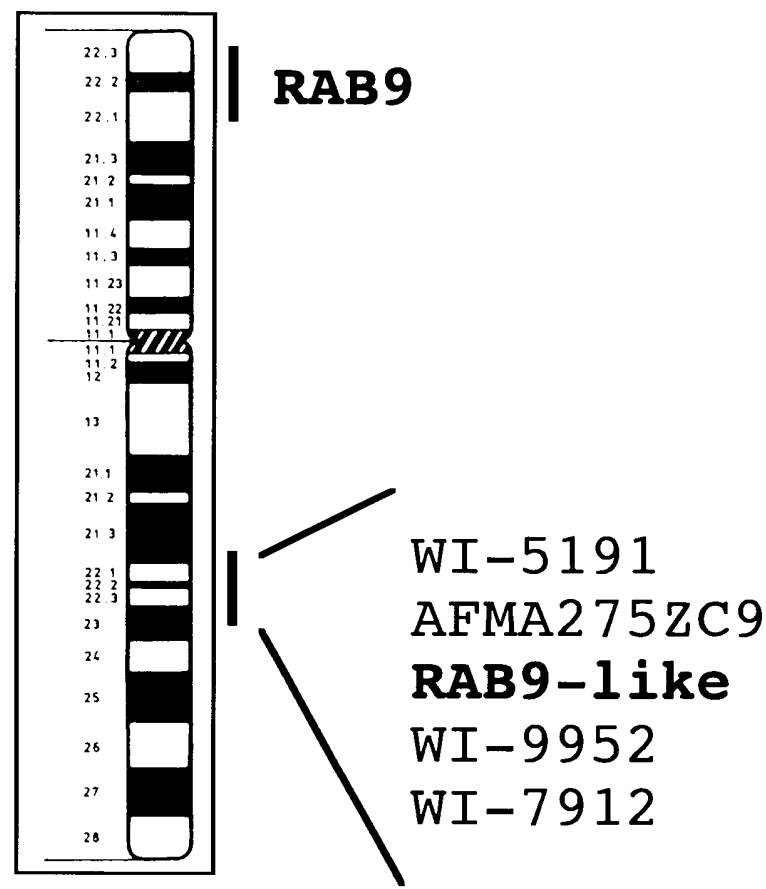

\section{Chromosome X}

Fig. 4. Chromosomal placement of human RAB9-like and $R A B 9$ genes at a relative distance to framework markers on the WICGR (Whitehead Institute/MIT Center of Genome Research) radiation hybrid map of the human genome. The approximate corresponding cytogenetic locations of the RAB9-like and RAB9 genes are on human chromosome Xq22 and Xp22.3 regions, respectively

The data described in this present report may serve as tools for the investigation of mammalian $R A B$ genes and function.

Acknowledgments We thank all the personnel contributing to the human genome sequencing project at the Sanger Center for their generous providsion of all the sequencing data available in the public arena.

\section{References}

Barbosa MD, Nguyen QA, Tchernev VT, Ashley JA, Detter JC, Blaydes SM, Brandt SJ, Chotai D, Hodgman C, Solari RC, Lovett M, Kingsmore SF (1996) Identification of the homologous beige and Chediak-Higashi syndrome genes. Nature 382:262-265

Barbosa MD, Barrat FJ, Tchernev VT, Nguyen QA, Mishra VS, Colman SD, Pastural E, Dufourcq-Lagelouse R, Fischer A, Holcombe RF, Wallace MR, Brandt SJ, de Saint Basile G, 
Kingsmore SF (1997) Identification of mutations in two major mRNA isoforms of the Chediak-Higashi syndrome gene in human and mouse. Hum Mol Genet 6:1091-1098

Brandt EJ, Elliott RW, Swank RT (1975) Defective lysosomal enzyme secretion in kidneys of Chediak-Higashi (beige) mice. J Cell Biol 67:774-788

Burkhardt JK, Wiebel FA, Hester S, Argon Y (1993) The giant organelles in beige and Chediak-Higashi fibroblasts are derived from late endosomes and mature lysosomes. J Exp Med 178:1845-1856

Chavrier P, Parton RG, Hauri HP, Simons K, Zerial M (1990) Localization of low molecular weight GTP binding proteins to exocytic and endocytic compartments. Cell 62:317-329

Chavrier P, Goud B (1999) The role of ARF and Rab GTPases in membrane transport. Curr Opin Cell Biol 11:466-475

Davies JP, Cotter PD, Ioannou YA (1997) Cloning and mapping of human Rab7 and Rab9 cDNA sequences and identification of a Rab9 pseudogene. Genomics 41:131-134

Gonzalez L Jr, Scheller RH (1999) Regulation of membrane trafficking: structural insights from a Rab/effector complex. Cell 96:755-758

Lombardi D, Soldati T, Riederer MA, Goda Y, Zerial M, Pfeffer SR (1993) Rab9 functions in transport between late endosomes and the trans Golgi network. EMBO J 12:677-682

Miyajima N, Burge CB, Saito T (2000) Computational and experimental analysis identifies many novel human genes. Biochem Biophys Res Commun 272:801-807

Novick P, Zerial M (1997) The diversity of Rab proteins in vesicle transport. Curr Opin Cell Biol 9:496-504

Nuoffer C, Balch WE (1994) GTPases: multifunctional molecular switches regulating vesicular traffic. Annu Rev Biochem 63:949-990
Riederer MA, Soldati T, Shapiro AD, Lin J, Pfeffer SR (1994) Lysosome biogenesis requires Rab9 function and receptor recycling from endosomes to the trans-Golgi network. J Cell Biol 125:573-582

Schmitt HD, Wagner P, Pfaff E, Gallwitz D (1986) The ras-related YPT1 gene product in yeast: a GTP-binding protein that might be involved in microtubule organization. Cell 7:401-412

Seki N, Sugano S, Suzuki Y, Nakagawara A, Ohira M, Muramatsu M, Saito T, Hori T (1998) Isolation, tissue expression, and chromosomal assignment of human RGS5, a novel G-protein signaling regulator gene. J Hum Genet 43:202-205

Seki N, Hattori A, Hayashi A, Kozuma S, Sasaki M, Suzuki Y, Sugano S, Muramatsu MA, Saito T (1999) Cloning and expression profile of mouse and human genes, Rnf11/RNF11, encoding a novel RING-H2 finger protein. Biochim Biophys Acta 1489:421-427

Seki N, Yoshikawa T, Hattori A, Miyajima N, Muramatsu M, Saito T (2000) cDNA cloning of a human RAB26-related gene encoding a Ras-like GTP-binding protein on chromosome 16p13.3 region. J Hum Genet 45:309-314

Shapiro AD, Riederer MA, Pfeffer SR (1993) Biochemical analysis of rab9, a ras-like GTPase involved in protein transport from late endosomes to the trans Golgi network. J Biol Chem 268:69256931

Willingham MC, Spicer SS, Vincent RA Jr (1981) The origin and fate of large dense bodies in beige mouse fibroblasts. Lysosomal fusion and exocytosis. Exp Cell Res 136:157-168

Zahraoui A, Touchot N, Chardin P, Tavitian A (1989) The human Rab genes encode a family of GTP-binding proteins related to yeast YPT1 and SEC4 products involved in secretion. J Biol Chem 264: 12394-12401 